\title{
A theoretical and experimental investigation of smooth- and wavy ice layers in laminar and turbulent flow inside an asymmetrically cooled parallel-plate channel
}

\author{
B. Weigand, H. Beer, Darmstadt, Germany
}

\begin{abstract}
The present paper shows the adaption of the numerical model originally developed by Weigand and Beer [14] for calculating steady-state ice layers inside an asymmetrically cooled parallel-plate channel. The investigation shows the characteristics in ice formation behaviour due to asymmetrically cooled walls. Further, a simple analytical model is presented for calculating smooth ice layers in turbulent flow. The study is supported by own measurements of the freezing fronts inside an asymmetrically cooled channel. A comparison between theoretical calculations and measurements shows generally good agreement.
\end{abstract}

Eine theoretische und experimentelle Untersuchung von glatten und welligen Eisschichten in laminarer und turbulenter Strömung in einem ebenen, asymmetrisch gekühlten Kanal

Zusammenfassung. Die vorliegende Arbeit beschreibt die Anwendung des von Weigand und Beer [14] entwickelten, numerischen Modells zur Vorhersage von Eisschichten in einem ebenen, asymmetrisch gekühlten Kanal. Die Studie befaßt sich mit den Unterschieden in der Eisschichtbildung aufgrund der asymmetrisch gekühlten Kanalwände. Weiterhin wird ein einfaches Verfahren angegeben, mit dem sich die Gestalt von glatten Eisschichten bei turbulenter Strömung und asymmetrischer Kühlung sehr einfach berechnen läßt. Die analytisch und numerisch gewonnenen Resultate werden anschließend mit eigenen Messungen yon Eisschichten verglichen, wobei eine im allgemeinen gute Übereinstimmung zwischen Theorie und Experiment zu beobachten ist.

$\begin{array}{ll}\text { Nomenclature } \\ A^{+} \quad \text { damping function } \\ a & \text { thermal diffusivity } \\ B & \text { dimensionless freezing parameter: } \theta_{c} k_{\mathrm{s}} / k_{\mathrm{L}} \\ F & \text { modified stream function } \\ H & \text { distance between parallel plates } \\ K & \text { acceleration parameter: }\left(v_{\mathrm{L}} / \bar{u}^{2}\right) \mathrm{d} \bar{u} / \mathrm{d} x \\ k & \text { thermal conductivity } \\ l & \text { mixing length } \\ p & \text { pressure } \\ P r & \text { Prandtl number: } v_{\mathrm{L}} / a_{\mathrm{L}} \\ P r_{\mathrm{t}} & \text { turbulent Prandt number: } \varepsilon_{m} / \varepsilon_{\mathrm{h}} \\ R_{2 H} & \text { Reynolds number based on } 2 H: \bar{u}_{0} 2 H / v_{\mathrm{L}} \\ T & \text { temperature } \\ T_{\mathrm{F}} & \text { freezing temperature of the liquid } \\ T_{0} & \text { constant inlet temperature of the liquid } \\ T_{\mathrm{w}} & \text { wall temperature of the lower wall } \\ u, v & \text { velocity components }\end{array}$

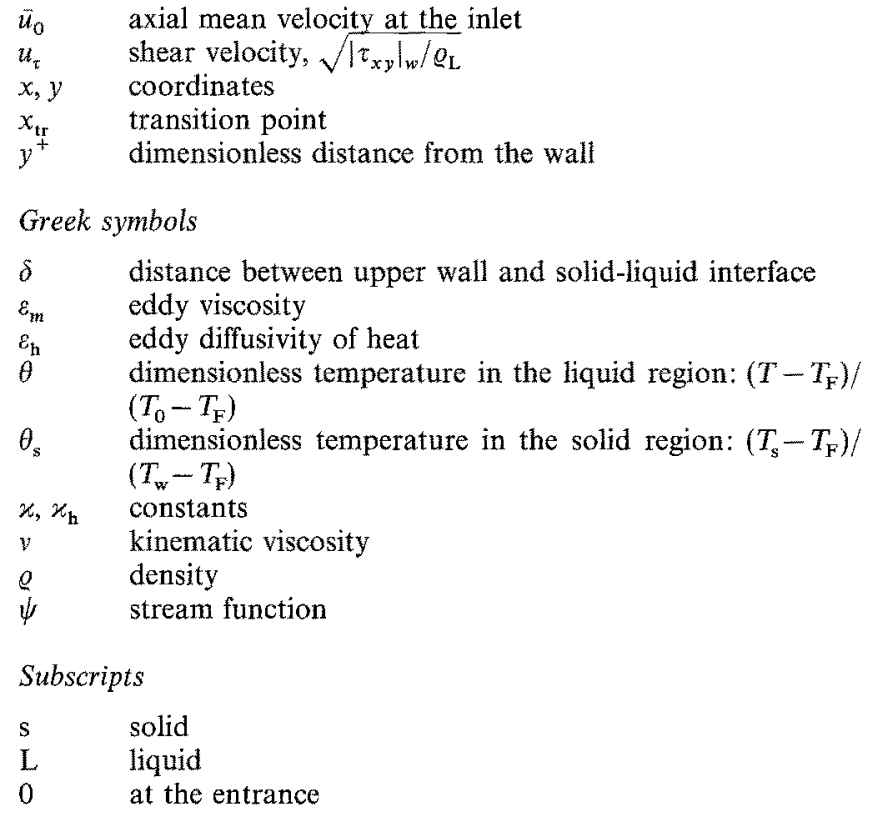

\section{Introduction}

Internal ice formation phenomena can be observed in many industrial processs, such as the freezing of flowing water in pipes or rectangular channels, the blockage of chemical process lines and the freezing of liquid metals in many casting operations, where molten material is poured through channels and nozzles, the walls of which are initially below the freezing temperature of the flowing material.

Many theoretical and experimental studies have been performed for fluid flow with solidification in circular tubes and parallel plate channels and its effect upon laminar flow heat transfer $[1-5]$. Also for the case of smooth ice layers in turbulent flow some investigations are known [6-8].

Since 1979 it is known that smooth ice layers in turbulent flow inside cooled pipes are only present for low and moderate cooling parameters. If the wall cooling exceeds some 
critical value, for a fixed Reynolds number, a strong interaction between the increasing ice layer thickness in flow direction and the turbulent flow takes place. These interactions result in a flow laminarization near the entrance of the chilled region. The flow laminarization leads to the development of wacy ice layers in turbulent flow. The seminal papers in this field are due to Gilpin [9], [10] who observed wavy ice layers appearing inside a cooled pipe subjected to turbulent internal flow. The wavy ice structures developed without an initial perturbation of the frozen crust. On the other hand, however, the ice-layer on a flat plate had to be perturbed by melting a heated cylinder half a diameter into the ice in order to get wavy ice layers. Without doing this, only one wave could be observed in the frozen crust on a flat plate [11]. The reason for the appearance of one wave in the ice layer on a flat plate is found to be the transition of the flow from laminar to turbulent state and not laminarization effects within the flow.

Seki et al. [12] and later Weigand and Beer [13] performed experiments in a parallel-plate channel with both walls kept at the same uniform temperature, which was below the freezing temperature of the flowing water. Because Seki et al. [12] investigated only a small range of Reynolds numbers and cooling parameters, they were able to observe only one wave in the ice. Weigand and Beer [13] showed the presence of many waves in the cooled channel which developed without perturbing the frozen crust. Therefore, the ice formation behaviour in the symmetrically cooled channel is found to be quite similar to the one in a cooled pipe.

For the case of ice layers with one wave Weigand and Beer [14] developed a numerical model for predicting the distribution of the frozen crust in a symmetrically cooled channel. Their numerical results agree well with experimental findings.

On the other hand freezing phenomena inside an asymmetrically cooled channel are not well investigated up to now. For the case of laminar internal flow only the study of Cheng and Sum-Lok Wong [5] is known in literature, which is an analytical approximative investigation. No numerical calculations were performed for the case of laminar flow in an asymmetrically cooled channel. For the case of turbulent flow only the two experimental studies [15] and [16] exist in literature. The major concern of Tago et al. [15] was the measurement of the velocity profiles and the turbulence intensity in the flow along a developing ice-layer surface, but solely at flow-rate Reynolds number $R e_{2 H}$ of approximately 12,000 . Weigand and Beer [16] presented an experimental study of the morphology of ice structure in an asymmetrically cooled channel. The found out that wavy ice layers with many waves developed without perturbing the frozen crust, but that the stability of the freezing fronts are more or less independent of the Reynolds number. This independence of the stability of the frozen crust from the Reynolds number is characteristic for a freezing front on a flat plate [11], but not for the behaviour of wavy ice layers in a symmetrically cooled channel. Therefore, the development and the be- haviour of the ice layers in an asymmetrically cooled channel must be understood as a connecting link between wavy ice layers caused by flow laminarization (symmetrically cooled channel) and wavy ice layers caused by the transition from laminar to turbulent flow (flat pate).

Hence, the present investigation has some different requests. One is to check the accuracy and the limitations of the approximative calculation method given in [5] against a numerical calculation of the frozen crust. Also, there is a need for the development of a model for predicting smooth ice layers in turbulent flow in an asymmetrically cooled channel, because there exists none in literature. The third demand of this paper is to examine whether it is possible to use the model from [14] for calculating wavy ice layers with one wave in turbulent flow. This aspect is very interesting because of the above mentioned differences in the behaviour of the freezing fronts and in respect to the key factors which are responsible for the shape of the frozen crusts. The present theoretical investigation is supported by measurements presented in [16] and some others obtained in the meantime.

\section{Physical mechanism and classification of ice-structures}

Figure 1 shows the geometrical configuration and the coordinate system. The flow enters the chilled region at $x=0$ with a fully developed velocity profile and with a constant inlet temperature $T_{0}$. In the cooling section, the temperature of the lower wall is maintained at a constant value $T_{\mathrm{w}}$, which is lower than the freezing temperature $T_{\mathrm{F}}$ of the fluid and therefore, a frozen layer is generated at the lower cooled wall. The upper wall of the channel is perfectly isolated.

All the different shapes of steady state ice layers, appearing in the asymmetric cooled channel, can be classified according to Weigand and Beer [16] with the help of a $\theta_{c}-\operatorname{Re}_{2 H}$ diagram, which is shown in Fig. 2. The dimensionless temperature ratio $\theta_{c}$ and the Reynolds number $R e_{2 H}$ are defined by

$\theta_{c}=\frac{T_{\mathrm{w}}-T_{\mathrm{F}}}{T_{\mathrm{F}}-T_{0}} ; \quad R e_{2 H}=\frac{\bar{u}_{0} 2 H}{\nu_{\mathrm{L}}}$

where $\bar{u}_{0}$ denotes the axial mean velocity at the entrance of the test section. Figure 3 shows some photographs of different types of ice layers which could be observed in experiment. The scaling in the photographs is given in $\mathrm{cm}$. For low

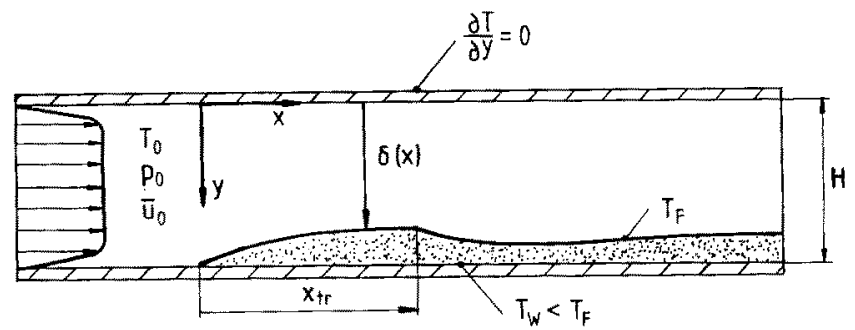

Fig. 1. Physical model and coordinate system 


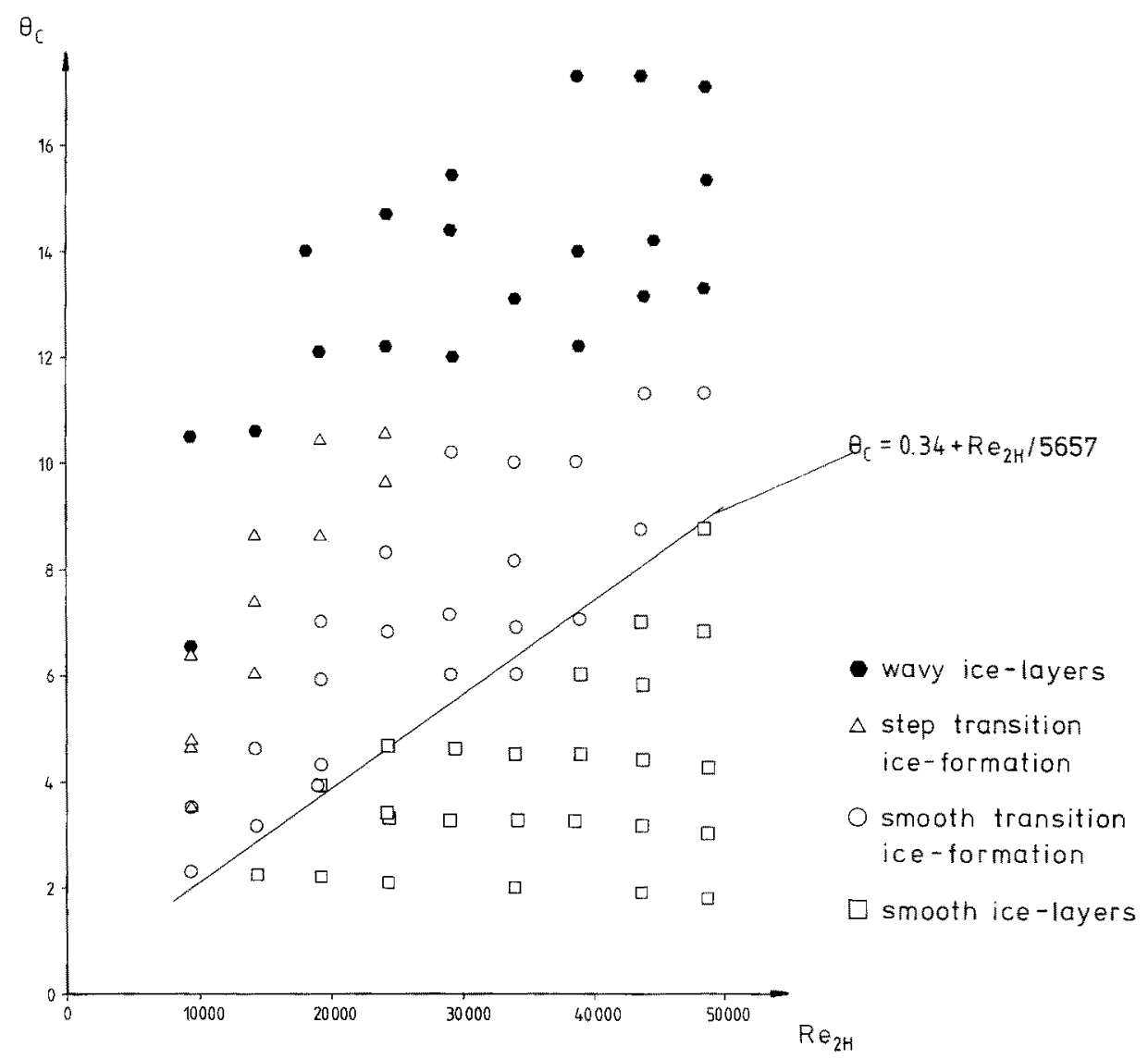

Fig. 2. Classification of different freezing phenomena in a $\theta_{c}-R e_{2 H}$ coordinate system values of $\theta_{c}$ a smooth ice layer can be observed. This type of ice layer is generally very thin (see Fig. 3). Therefore, the shape of the frozen crust doesn't effect the flow characteristics and the turbulence structure of the main flow. Increasing the value of $\theta_{c}$, while the Reynolds number remains fixed, leads to flow laminarization near the entrance of the test section. This is due to the strong acceleration of the main flow caused by the increasing thickness of the frozen crust in flow direction. It can be observed that the acceleration parameter $K$ defined as

$K=\frac{v_{\mathrm{L}}}{\bar{u}^{2}} \frac{\mathrm{d} \bar{u}}{\mathrm{~d} x}=-\frac{2}{R e_{2 H}} \frac{\mathrm{d} \delta}{\mathrm{d} x}$

attaines values greater than $K_{\text {crit }}=2 \cdot 10^{-6}-3 \cdot 10^{-6}$ near the entrance of the chilled region. $K_{\text {crit. }}$ denotes the critical value of the acceleration parameter which is sufficient to relaminarize an initially turbulent flow (see Moretti and Kays [17]). In a distance from the entrance the free channel height $\delta$ reaches a nearly constant value $(K \rightarrow 0)$ and hence, the fluid recedes to its initially turbulent state. This results in an increasing heat flux from the fluid to the solid crust and therefore, in a decreasing ice layer thickness in this region. The above mentioned mechanism leads to the development of a wave in the frozen crust. A more detailed explanation of the phenomena can be found in [14]. It can be easily understood that the difference in ice layer thickness between the wave crest and the wave trough primarely depends on the difference in heat transfer between the laminarized state of the flow near the entrance of the cooling section and the fully turbulent state of the flow. Therefore, increasing the dimensionless temperature ratio $\theta_{c}$ for a constant value of $R e_{2 H}$ leads to more pronounced waves in the crust because of an intensified laminarization of the flow in the entrance region of the chilled section. For $\theta_{c}>12$ the ice layer is found to be unstable against finite perturbations and a wavy ice structure with many waves can be observed. It is interesting to note that this value of $\theta_{c}=12$ depends only slightly on the value of the Reynolds number. This observation is in contrast to the case of a symmetrically cooled channel [14], but in accordance with experiments which were carried out for a cooled flat plate [11]. For a more detailed description of the experiments and the physical phenomena the reader is refered to [16].

\section{Analysis}

3.1 A numerical model for the calculation of smooth ice layers and ice layers with one wave

The following numerical model is analogous to the one used by the authors [14] for calculating ice layers inside a symmetrically cooled channel. Therefore, the model is only discussed 


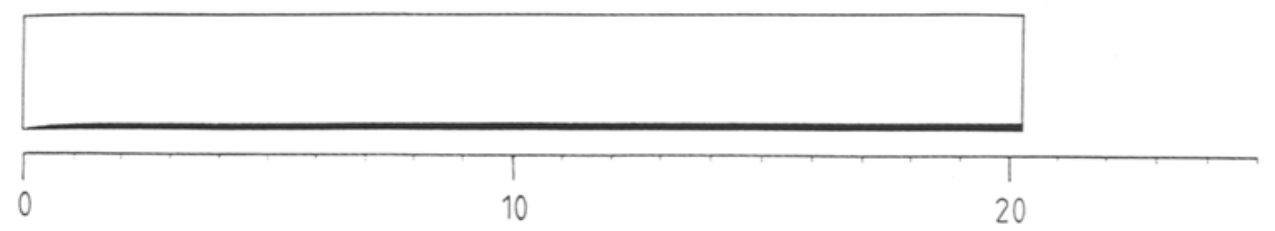

Smooth ice layer $\left(\operatorname{Re}_{2 H}=34000, B=18\right)$

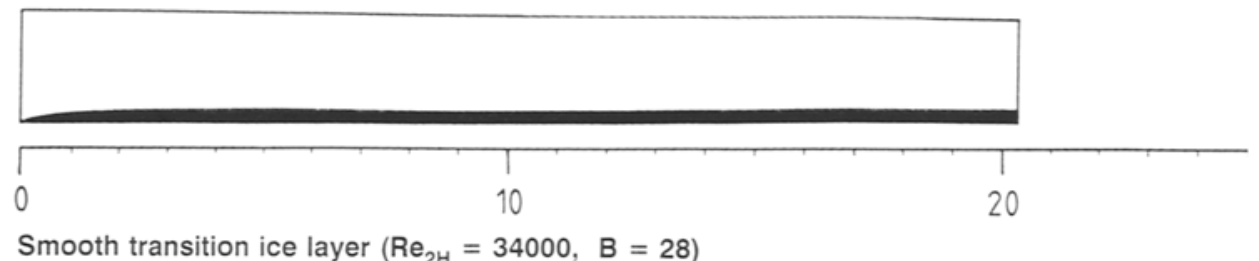

Smooth transition ice layer $\left(\mathrm{Re}_{2 \mathrm{H}}=34000, \quad \mathrm{~B}=28\right)$

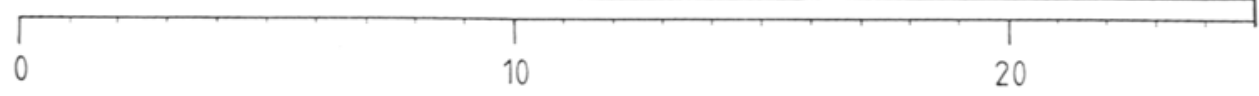

Step transition ice layer $\left(\mathrm{Re}_{2 \mathrm{H}}=14000, \mathrm{~B}=24\right)$

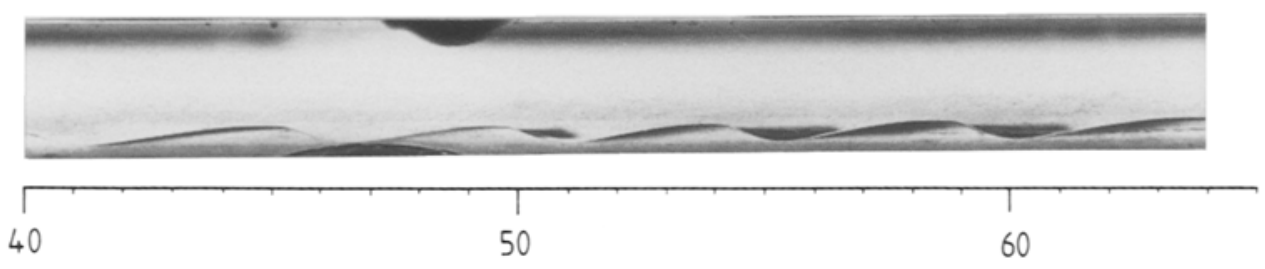

Wavy ice layer $\left(\mathrm{Re}_{2 \mathrm{H}}=44000, \mathrm{~B}=68\right.$ )

Fig. 3. Different shapes of ice layers in turbulent flow

briefly in the present paper. For more detailed informations concerning the model, the reader is refered to [14].

By assuming an incompressible, Newtonian fluid with constant fluid properties, the steady state conservation equations for the fluid can be written in the following form by introducing a stream function, defined by

$u^{*}=-\frac{\partial \psi^{*}}{\partial y^{*}}, \quad v^{*}=\frac{\partial \psi^{*}}{\partial \tilde{x}}, \quad F=\psi^{*} / \tilde{\delta}$

into the conservation equations. This results in

$$
\begin{aligned}
& \frac{1}{\tilde{\delta}^{2}} \frac{\partial}{\partial \tilde{y}}\left[\left(1+\varepsilon_{m}^{*}\right) \frac{\partial^{2} F}{\partial \tilde{y}^{2}}\right] \\
& =\frac{\partial p^{*}}{\partial \tilde{x}}+\frac{\partial F}{\partial \tilde{y}} \frac{\partial^{2} F}{\partial \tilde{x} \partial \tilde{y}}-\frac{\partial F}{\partial \tilde{x}} \frac{\partial^{2} F}{\partial \tilde{y}^{2}}-\frac{1}{\tilde{\delta}} \frac{\mathrm{d} \tilde{\delta}}{\mathrm{d} \tilde{x}} F \frac{\partial^{2} F}{\partial \tilde{y}^{2}} \\
& 0=\frac{\partial p^{*}}{\partial \tilde{y}} \\
& \frac{1}{\tilde{\delta}^{2}} \frac{\partial}{\partial \tilde{y}}\left[\left(1+\frac{P r}{P r_{\mathrm{t}}} \varepsilon_{m}^{*}\right) \frac{\partial \theta}{\partial \tilde{y}}\right] \\
& =\operatorname{Pr}\left[\frac{\partial F}{\partial \tilde{y}} \frac{\partial \theta}{\partial \tilde{x}}-\frac{\partial F}{\partial \tilde{x}} \frac{\partial \theta}{\partial \tilde{y}}-\frac{1}{\tilde{\delta}} \frac{\mathrm{d} \tilde{\delta}}{\mathrm{d} \tilde{x}} F \frac{\partial \theta}{\partial \tilde{y}}\right]
\end{aligned}
$$

with the dimensionless quantities

$$
\begin{aligned}
& y^{*}=\frac{y}{H}, \quad \tilde{x}=\frac{x}{H}, \quad \tilde{y}=\sqrt{\operatorname{Re}_{H}}\left(1-\frac{y}{\delta}\right), \quad \tilde{\delta}=\frac{\delta}{H}, \quad u^{*}=\frac{u}{\bar{u}_{0}} \\
& v^{*}=\frac{v}{\bar{u}_{0}} \sqrt{\operatorname{Re}_{H}}, \quad p^{*}=\frac{p}{\varrho_{\mathrm{L}} \bar{u}_{0}^{2}}, \quad \operatorname{Pr}=\frac{v_{\mathrm{L}}}{a_{\mathrm{L}}}, \quad \operatorname{Pr} r_{\mathrm{t}}=\frac{\varepsilon_{m}^{*}}{\varepsilon_{\mathrm{l}}^{*}}, \varepsilon_{m}^{*}=\frac{\varepsilon_{m}}{v_{\mathrm{L}}} \\
& \epsilon_{\mathrm{h}}^{*}=\frac{\varepsilon_{\mathrm{h}}}{v_{\mathrm{L}}}, \quad \theta=\frac{T-T_{\mathrm{F}}}{T_{0}-T_{\mathrm{F}}}, \quad \psi^{*}=\frac{\psi}{\bar{u}_{0} H}, \quad R e_{H}=\frac{\bar{u}_{0} H}{v_{\mathrm{L}}}
\end{aligned}
$$

and the eddy viscosity $\varepsilon_{m}$ defined in the usual way

$$
-\varrho_{\mathbf{L}} \overline{u^{\prime} v^{\prime}}=\varrho_{\mathbf{L}} \varepsilon_{m} \frac{\partial u}{\partial y} \text {. }
$$

By deriving the Eqs. (4)-(6) the usual boundary layer assumptions were made, which are a common treatment of the conservation equations for channel flows [18]. The boundary conditions belonging to the set of Eqs. (4)-(6) are given by

$$
\begin{array}{llll}
\tilde{x}=0: & \frac{\partial F}{\partial \tilde{y}}=\text { given, } & p^{*}=p_{0}^{*}, & \theta=1 \\
\tilde{y}=0: & \frac{\partial F}{\partial \tilde{y}}=0, & F=0, & \theta=0
\end{array}
$$

$\tilde{y}=\sqrt{R \mathrm{e}_{H}}: F=\sqrt{R \mathrm{e}_{H}} / \tilde{\delta}, \quad \frac{\partial F}{\partial \tilde{y}}=0, \quad \frac{\partial \theta}{\theta \tilde{y}}=0$. 
Introducing the new coordinates $\tilde{x}, \tilde{y}$ into the conservation equations is very useful in order to solve the problem, because the duct with variable distance between the wall and the solid liquid interface is transformed into a duct with constant height. The conservation of mass in integral form results in an additional boundary condition for the stream function $F$ at $\tilde{y}=\sqrt{R e_{H}}$, as it can be seen from Eq. (9).

In addition to the conservation equations for the fluid, the energy equation for the solid region is required. By assuming constant properties in the solid phase and negligible axial heat conduction, the one dimensional heat conduction equation in the frozen crust can be integrated easily. The temperature distribution in the ice layer can be shown to be

$\theta_{\mathrm{s}}=\frac{T_{\mathrm{s}}-T_{\mathrm{F}}}{T_{\mathrm{w}}-T_{\mathrm{F}}}=\frac{y-\delta}{H-\delta} ; \quad \delta \leq y \leq H$.

The conservation equations for the liquid region, Eqs. (4)(6), are coupled with Eq. (10) by the interface energy equation

$k_{\mathrm{s}} \frac{\partial T_{\mathrm{s}}}{\partial y}=k_{\mathrm{L}} \frac{\partial T}{\partial y} ; \quad y=\delta(x)$

which states simply the fact that the heat transfered from the fluid to the solid crust is conducted through the solid layer. Equation (11) can be rewritten in dimensionless form by using Eq. (10) and Eq. (7). This results in the following expression for the unknown free channel height $\widetilde{\delta}(\tilde{x})$

$\tilde{\delta}(\tilde{x})=\left.\frac{\partial \theta}{\partial \tilde{y}}\right|_{\tilde{y}=0} \frac{\sqrt{\operatorname{Re}_{H}}}{B} /\left(1+\left.\frac{\sqrt{\operatorname{Re}_{H}}}{B} \frac{\partial \theta}{\partial \tilde{y}}\right|_{\tilde{y}=0}\right)$

where the dimensionless freezing parameter $B$ is given by

$B=\frac{k_{\mathrm{s}}}{k_{\mathrm{L}}} \frac{T_{\mathrm{F}}-T_{\mathrm{w}}}{T_{0}-T_{\mathrm{F}}}=\frac{k_{\mathrm{s}}}{k_{\mathrm{L}}} \theta_{c}$.

In order to solve the above described problem, the eddy viscosity, which appears in the momentum equation and in the energy equation for the liquid, has to be modelled. For the case of a smooth ice layer, a simple mixing length model was appropriate. The mixing length distribution was described by the well known mixing length formula of $\mathrm{Ni}$ kuradse, modified by a damping factor according to van Driest

$$
\begin{aligned}
& 0 \leq \tilde{y} \leq \sqrt{\operatorname{Re}_{H}^{-}} / 2: \\
& \tau=\frac{l}{\delta}=\frac{1}{2}\left(0.14-0.08\left(1-\frac{2 \tilde{y}}{\sqrt{\operatorname{Re}_{a}}}\right)^{2}\right. \\
& \left.-0.06\left(1-\frac{2 \tilde{y}}{\sqrt{\operatorname{Re}_{H}}}\right)^{4}\right)\left(1-\exp \left(y_{1}^{+} / A^{+}\right)\right) \\
& \sqrt{\operatorname{Re}_{H}} / 2 \leq \tilde{y} \leq \sqrt{\operatorname{Re}_{H}}: \\
& \widetilde{l}=\frac{l}{\delta}=\frac{1}{2}\left(0.14-0.08\left(1-\frac{2 \tilde{y}}{\sqrt{R_{\mathrm{e}_{H}}}}\right)^{2}\right. \\
& \left.-0.06\left(1-\frac{2 \tilde{y}}{\sqrt{R \mathrm{e}_{H}}}\right)^{4}\right)\left(1-\exp \left(y_{2}^{+} / A^{+}\right)\right)
\end{aligned}
$$

with the dimensionless wall distances

$y_{1}^{+}=y_{\mathrm{w} 1} u_{\tau 1} / v_{\mathrm{L}} ; \quad y_{2}^{+}=y_{\mathrm{w} 2} u_{\mathrm{\tau} 2} / v_{\mathrm{L}}$

where the index 1 indicates the solid liquid interface and 2 indicates the isolated wall.

The eddy viscosity is given in this case by

$\varepsilon_{m}^{*}=\tilde{\delta}\left(R e_{H}\right)^{3 / 2} \widetilde{T}^{2}\left|\frac{\partial^{2} F}{\partial \tilde{y}^{2}}\right|$

and $A^{+}$is a constant, in literature usually taken to be 26 .

For predicting wavy ice layers with one wave a more complicated turbulence model has to be used. This is because different flow regimes exist for this case in the cooled channel. We can devide the whole axial area into three distinct regions: For small values of the axial coordinate $\left(x<x_{t r}\right.$, region 1) the flow undergoes a rapid acceleration and flow laminarization can be observed. For large axial distances the free channel height is nearly constant and therefore, the flow behaviour in this region (region 3 ) is nearly the same as that in a parallel plate channel. In region 2 the flow changes from its laminarized state to a fully turbulent state, including a diffusor which enhances the turbulent fluctuations. Because we have used the turbulence model given in [14], only some differences as compared to the case of symmetrically cooled walls shall be pointed out. For a detailed description of the turbulence model in the case of symmetric ice formation once more the reader is refered to [14].

One empirical input which has to be made in the above mentioned turbulence model is the axial distance between the axial point where the acceleration parameter $K$, defined in Eq. (1), falls below its critical value $\left(K_{\text {crit }}=2 \cdot 10^{-6}\right)$ and the axial point $\tilde{x}_{\mathrm{tr}}$, where the ice-layer reaches its maximum thickness. This axial distance $\Delta \tilde{x}=\tilde{x}_{\mathrm{tr}}-\tilde{x}_{\mathrm{crit}}$ describes the axial region in which instabilitics in the laminar sublayer at the ice water interface develop in size. From measurements the following relation for $\Delta \tilde{x}$ could be derived

$\Delta \tilde{x}=1.4 \cdot 10^{7} \operatorname{Re}_{H}^{-3 / 2} B^{-0.4}$.

The functional relationship given by Eq. (17) is quite similar to the one given in [14].

In order to relate the eddy diffusivity of heat $\varepsilon_{\mathrm{h}}^{*}$, which appears in the energy equation, to the eddy viscosity $\varepsilon_{m}^{*}$, a formulation for the turbulent Prandtl number is required. In the present study as well as in [14], the turbulent Prandtl number concept according to Cebeci [18] was used.

The calculation of the frozen crust involves an iteration procedure. Initially a distribution of the ice layer thickness was assumed. With this variation of $\widetilde{\delta}(\tilde{x})$ the conservation Eqs. (4)-(6) were solved with the help of an implizit finitedifference method, which is known in literature as the Kellerbox method [18]. By solving the conservation equations for the present problem of a wavy ice layer with one wave, a highly dense grid has to be taken near the solid-liquid interface and near the upper isolated wall because of strong gradients appearing in this region. Additionally a very small 
grid spacing was used in the region of the wave. For calculating the velocity and the temperature distribution for the case of flow separation in the diffusor region, the FLARE approximation of Reyhner and Flügge-Lotz [19] was applied to represent small regions of separated flow. The use of boundary layer equations to represent separated flows presumes that the pressure gradient in normal direction and longitudinal diffusion are negligible in the region of recirculation. Also, it must be assumed that the region of recirculation remains small as compared to the characteristic length of the problem. In the present case, the error introduced by applying the FLARE approximation in the very small recirculation region can be neglected with good accuracy (see Kwon et al. [20]).

After solving the Eqs. (4)-(6) in conjunction with the boundary conditions, Eq. (9), a new distribution of $\tilde{\delta}(\tilde{x})$ can be calculated by inserting the yet known temperature gradient at the solid-liquid interface into Eq. (12). This iteration process is repeated until $\Delta \tilde{\delta}=\left|\tilde{\delta}^{(i)}-\tilde{\delta}^{(i-1)}\right|<0.01$ at every axial position for two successive iterations.

\subsection{A simple analytical model for predicting the shape of smooth ice layers}

The present model, which can be used for calculating steady state smooth ice layers for asymmetrically cooled walls, is quite similar to the approximation model of Shibani and Özisik [7] for the case of symmetric cooled walls.

We make the assumption that the fully turbulent velocity profile at the entrance of the test section is not affected by the growing ice layer. This means that the shape of the axial velocity distribution is given by

$\frac{u}{\bar{u}}=f\left(\frac{y}{\delta}\right) ; \bar{u}=\bar{u}_{0} \frac{H}{\delta}$.

The assumption of a functional relationship for the velocity profile according to Eq. (18) is consistent with the supposition that $\mathrm{d} \delta / \mathrm{d} x \rightarrow 0$. This assumption leads to very good results in the present case, because of the thin ice layers under consideration.

Inserting the velocity distribution given by Eq. (18) into the energy equation results in the following parabolic partial differential equation

$f(\eta) \frac{\partial \theta}{\partial \xi}=\frac{1}{R e_{H} P r} \frac{\partial}{\partial \eta}\left[\left(1+\frac{P r}{P r_{t}} \varepsilon_{m}^{*}\right) \frac{\partial \theta}{\partial \eta}\right]$

with the new variables

$\eta=\frac{y}{\delta} ; \quad \xi=\int_{0}^{\bar{x}} \frac{\mathrm{d} \bar{x}}{\tilde{\delta}(\bar{x})}$

and the boundary conditions

$$
\begin{aligned}
& \theta(0, \eta)=1 \\
& \left.\frac{\partial \theta}{\partial \eta}\right|_{\eta=0}=0, \quad \theta(\xi, 1)=0 .
\end{aligned}
$$

The eddy viscosity $\varepsilon_{m}^{*}$, appearing in Eq. (19), can be prescribed with the help of the mixing length model given by Eqs. (14)-(16). This results in the following expression:

$\varepsilon_{m}^{*}=\operatorname{Re}_{H} \tau^{2}\left|f^{\prime}(\eta)\right|$.

Equation (22) shows the fact that $\varepsilon_{m}^{*}$ is only a function of $\eta$ and not a function of the axial coordinate. This is because of the linear dependence of the mixing length on $\tilde{\delta}$, given by Eq. (14).

The turbulent Prandtl number, appearing in Eq. (19), was taken to be equal to 0.9 for simplicity. The solution of the partial differential Eq. (19) can be obtained easily by using the method of separation of variables. This results in the following expression for the temperature distribution

$\theta(\xi, \eta)=\sum_{n=1}^{\infty} C_{n} B_{n}(\eta) \exp \left(-\frac{\lambda_{n}^{2}}{\operatorname{Re}_{H} \operatorname{Pr}} \xi\right)$

with the constants $C_{n}$ given by

$C_{n}=-B_{n}^{\prime}(1) /\left(\lambda_{n}^{2} \int_{0}^{1} f(\eta) B_{n}^{2}(\eta) \mathrm{d} \eta\right)$.

The functions $B_{n}(\eta)$ are the solutions of the Sturm-Liouville problem corresponding to Eq. (19)

$\frac{\mathrm{d}}{\mathrm{d} \eta}\left[\left(1+\frac{P r}{P r_{\mathrm{t}}} \varepsilon_{m}^{*}\right) \frac{\mathrm{d} B_{n}}{\mathrm{~d} \eta}\right]+\lambda_{n}^{2} f(\eta) B_{n}=0$

with the boundary conditions

$\mathrm{d} B_{n} / \mathrm{d} \eta=0, \quad B_{n}(1)=0$

and the arbitrary normalizing condition that $B_{n}(0)=1$. The eigenfunctions and the eigenvalues of Eqs. (25)-(26) were calculated with the help of a Runge-Kutta method.

Inserting the temperature gradient, calculated from Eq. (23), into the interface energy Eq. (11) and using Eq. (10), the following expression for the free channel height $\tilde{\delta}(\xi)$ can be derived

$\tilde{\delta}(\xi)=\left[1+B / \sum_{n=1}^{\infty} C_{n} B_{n}^{\prime}(1) \exp \left(-\frac{\lambda_{n}^{2}}{R e_{H} P r} \xi\right)\right]^{-1}$.

The dependence of the integral coordinate $\xi$ on $\tilde{x}$ is given by Eq. (20). Table 1 shows eigenvalues $\lambda_{n}$ and the constants $C_{n} B_{n}^{\prime}(1)$ for three different Prandtl numbers and various values of the Reynolds number. Because the value of the Prandtl number for water is relatively high, it is sufficient to take only the first and the second eigenvalue and constant for calculating the shape of the frozen crust. Hence, the thickness of the ice layer can be predicted very easily by using Eq. (27).

For the case of a fully developed laminar flow entering the cooling section, the variation of the solidified crust can be calculated by setting $\varepsilon_{m}^{*}=0$ in the above mentioned equations. The problem of solidification of a laminar flowing liquid in an asymmetrically cooled channel submitted to external convection was solved by Cheng and Wong [5]. For 
Table 1. Eigenvalues and constants

\begin{tabular}{|c|c|c|c|c|c|c|}
\hline & \multicolumn{2}{|c|}{$\operatorname{Pr}=7$} & \multicolumn{2}{|c|}{$\operatorname{Pr}=10$} & \multicolumn{2}{|c|}{$\operatorname{Pr}=13$} \\
\hline \multicolumn{7}{|c|}{$R e_{2 H}=20,000$} \\
\hline$n$ & $\lambda_{n}$ & $C_{n} B_{n}^{\prime}(1)$ & $\lambda_{n}$ & $C_{n} B_{n}^{\prime}(1)$ & $\lambda_{n}$ & $C_{n} B_{n}^{\prime}(1)$ \\
\hline 1 & 8.53 & 72.27 & 9.23 & 84.68 & 9.74 & 94.52 \\
\hline 2 & 41.44 & 8.39 & 48.97 & 7.86 & 55.43 & 7.42 \\
\hline 3 & 83.48 & 3.31 & 99.28 & 3.11 & 112.85 & 2.94 \\
\hline 4 & 116.62 & 2.68 & 138.76 & 2.54 & 157.78 & 2.41 \\
\hline \multicolumn{7}{|c|}{$R e_{2 H}=40,000$} \\
\hline$n$ & $\lambda_{n}$ & $C_{n} B_{n}^{\prime}(1)$ & $\lambda_{n}$ & $C_{n} B_{n}^{\prime}(1)$ & $\lambda_{n}$ & $C_{n} B_{n}^{\prime}(1)$ \\
\hline 1 & 11.43 & 129.84 & 12.39 & 152.70 & 13.10 & 170.84 \\
\hline 2 & 55.82 & 14.54 & 66.03 & 13.69 & 74.79 & 12.96 \\
\hline 3 & 112.99 & 5.35 & 134.50 & 5.01 & 152.95 & 4.73 \\
\hline 4 & 158.78 & 3.99 & 189.15 & 3.73 & 215.23 & 3.51 \\
\hline \multicolumn{7}{|c|}{$R e_{2 H}=60,000$} \\
\hline n & $\lambda_{n}$ & $C_{n} B_{n}^{\prime}(1)$ & $\lambda_{n}$ & $C_{n} B_{n}^{\prime}(1)$ & $\lambda_{n}$ & $C_{n} B_{n}^{\prime}(1)$ \\
\hline 1 & 13.59 & 183.59 & 14.74 & 216.39 & 15.60 & 242.45 \\
\hline 2 & 66.58 & 20.24 & 78.79 & 19.14 & 89.27 & 18.15 \\
\hline 3 & 134.88 & 7.30 & 160.59 & 6.84 & 182.67 & 6.46 \\
\hline 4 & 189.67 & 5.29 & 226.04 & 4.92 & 257.26 & 4.64 \\
\hline
\end{tabular}

the case of very high values of the Biot number eigenvalues, constants and the shape of the frozen crust were compared with the results given in [5] and good agreement was found.

\section{Results and discussion}

\subsection{Smooth ice layers for laminar flow}

In Fig. 4 ice layers are plotted as a function of the axial coordinate. In this figure $(1-\delta / H)=0$ denotes the cooled lower wall of the channel and $(1-\delta / H)=1$ denotes the isolated upper wall. The figure shows a comparison of smooth ice layers for $R e_{2 H}=2000$ and $P r=10$, calculated with the numerical method presented in Chap. 3.1 and ice layers, calculated with the approximation method shown in the preceding chapter. In both methods the eddy viscosity $\varepsilon_{m}^{*}$ was taken to be zero and the velocity profile at the entrance of the cooling section was given by a Hagen-Poiseuille profile. It is obvious that the approximate solution for $\tilde{\delta}$ agrees very well with the numerical prediction up to $B=8$. Therefore, the simple approximation given by [5] can be used for calculating the shape of the freezing fronts, avoiding a numerical treatment of the conservation equations for this special case.

\subsection{Smooth ice layers for turbulent flow}

Figure 5 shows the variation of the ice layer thickness for a fixed value of the freezing parameter $B$ and a given value of the Prandtl number. It can be seen that increasing the Reynolds number leads to a decreasing thickness of the frozen crust. This is due to the enhanced heat transfer from the fluid to the solid-liquid interface with growing values of the Reynolds number. Figure 6 elucidates the effect of an

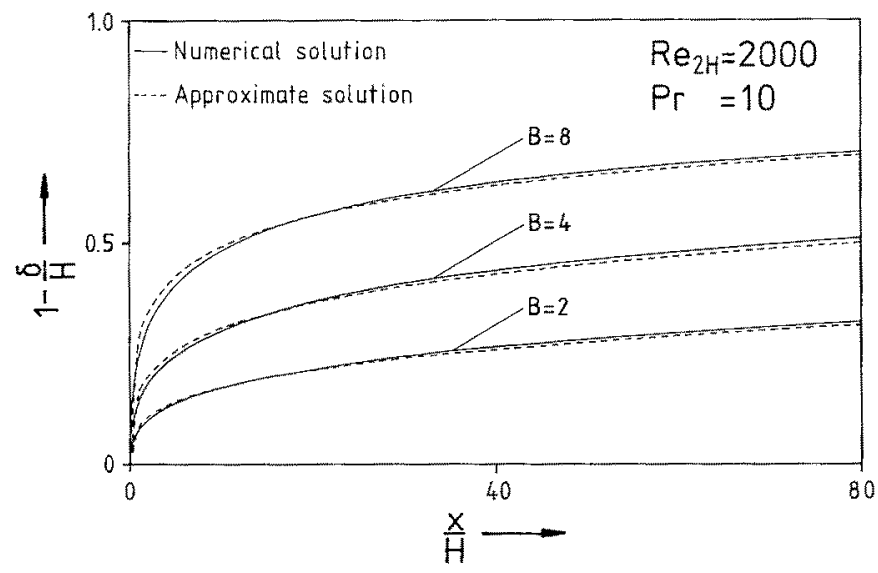

Fig. 4. Ice layers for laminar flow (smooth ice layers)

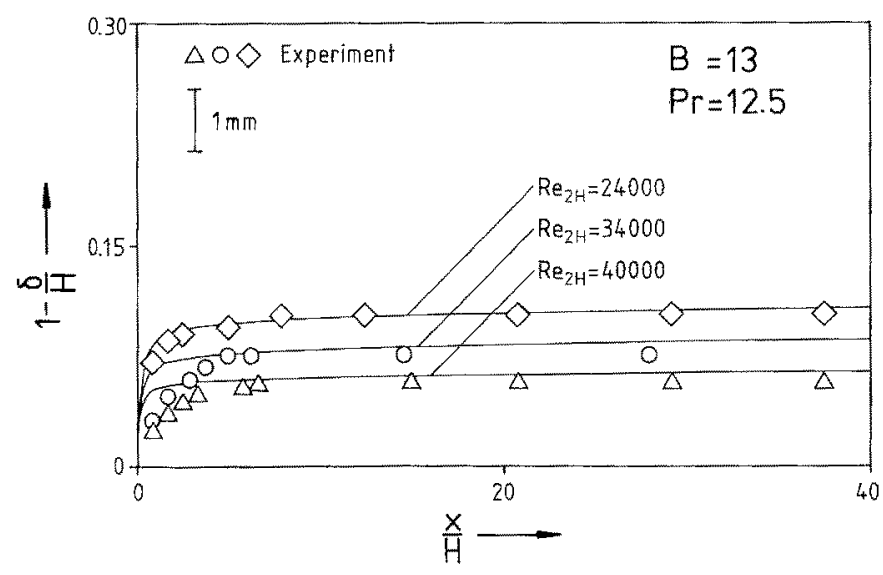

Fig. 5. Effect of Reynolds number on the axial distribution of the ice layer thickness for turbulent flow (smooth ice layers)

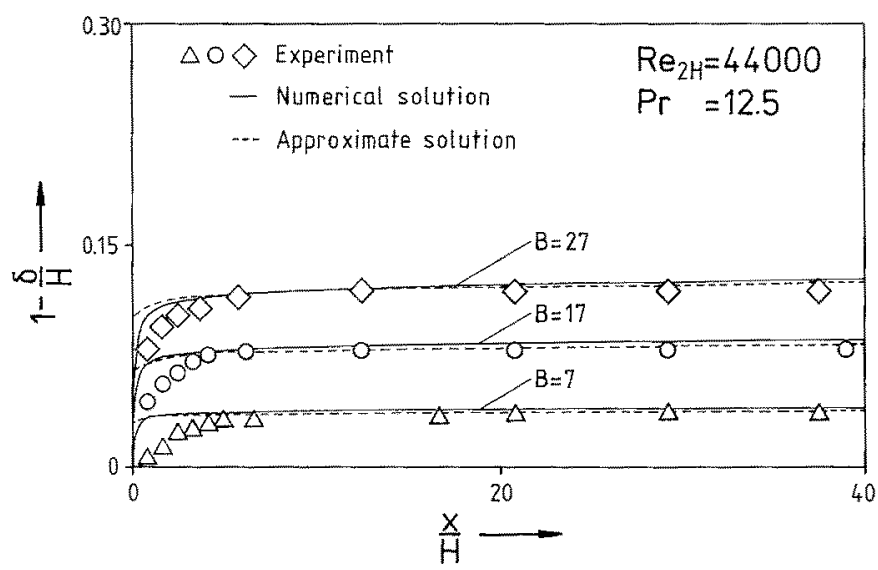

Fig. 6. Effect of $B$ on the axial distribution of the ice layer thickness for turbulent flow (smooth ice layers)

increasing cooling parameter $B$ for a constant value of the Reynolds number and a constant value of $P r$. It can be seen that increasing the cooling parameter $B$ leads to thicker ice layers. Both plots compare numerical calculations with measurements. It can be observed that the agreement between the numerical calculation and the experiment is quite well, 
especially if one realizes that the ice layers are extremely thin. Figure 5 shows a scale for $H=24 \mathrm{~mm}$ which gives an impression of the thickness of the frozen crust in reality. Figure 6 shows also comparisons between numerically calculated ice layers and ice layers predicted by the analytical approximation method. The differences which can be observed between the numerical calculations and the approximate solutions are mainly caused by the different turbulent Prandtl number concepts and by the prescribed error bound for $\left|\widetilde{\delta}^{(i)}-\widetilde{\delta}^{(i-1)}\right|$ applied to the numerical calculations.

From Figs. 5-6 it can be observed that more pronounced differences between the calculated ice layer thickness and the measurements can be detected for $0 \leq \tilde{x} \leq 4$. This is mainly because of experimental problems arising by measuring the thickness of the ice layers in this region.

Hence, the shape of smooth ice layers in turbulent flow can be calculated precisely by using the simple analytical approximation method presented in Chap. 3.2.

\subsection{Wavy ice layers with one wave in turbulent flow}

Figures 7 and 8 show comparisons between calculated and measured ice layers plotted as a function of the axial coordinate. Figure 7 elucidates that an increasing cooling parameter $B$ results in thicker ice layers for a given value of the Reynolds number. Further, the plots visualize that an increasing value of the cooling parameter $B$ tends to destabilize the ice layer for a fixed value of the Reynolds number. This can also be observed in Fig. 2. Figure 8 elucidates the effect of a variation of the Reynolds number for two different values of the cooling parameter $B$. It is obvious that an increasing Reynolds number causes a decreasing ice layer thickness. Therefore, increasing the Reynolds number for a fixed value of $B$ tends to stabilize the ice layer (see also Fig. 2). As it can be observed in Fig. 7 and Fig. 8, the agreement between numerical calculations and experiments is generally quite good. However, near the axial position where the ice layer reaches its minimum thickness, larger deviations can be observed. This can be attributed to three-dimensional effects appearing in the diffusor region and also to the simple turbulence model used in the present paper, which is not able to deal correctly with the extremely complex transitional flow, incorporating recirculation zones in the diffusor region.

For small waves in the ice layer the model is able to predict the shape of the frozen crust very precisely (Fig. 7 : $R e_{2 H}=34,000, \quad B=24 ; \quad R e_{2 H A}=24,000, \quad B=33 ; \quad$ Fig. 8: $B=28, \operatorname{Re}_{2 H}=24,000$ and $R e_{2 H}=34,000$ ).

Additionally, it should be recognized that for $R e_{2 H I}=$ $14,000, B=28$ the theoretical results deviate from the measurements for $\tilde{x}<\tilde{x}_{\mathrm{tr}}$. This signifies that a full laminarization of the flow has taken place in the entrance region of the cooled channel. In such a case, the shape of the ice layer for $\tilde{x}<\tilde{x}_{t r}$ can be calculated with good accuracy by neglecting the eddy viscosity in the conservation equations.

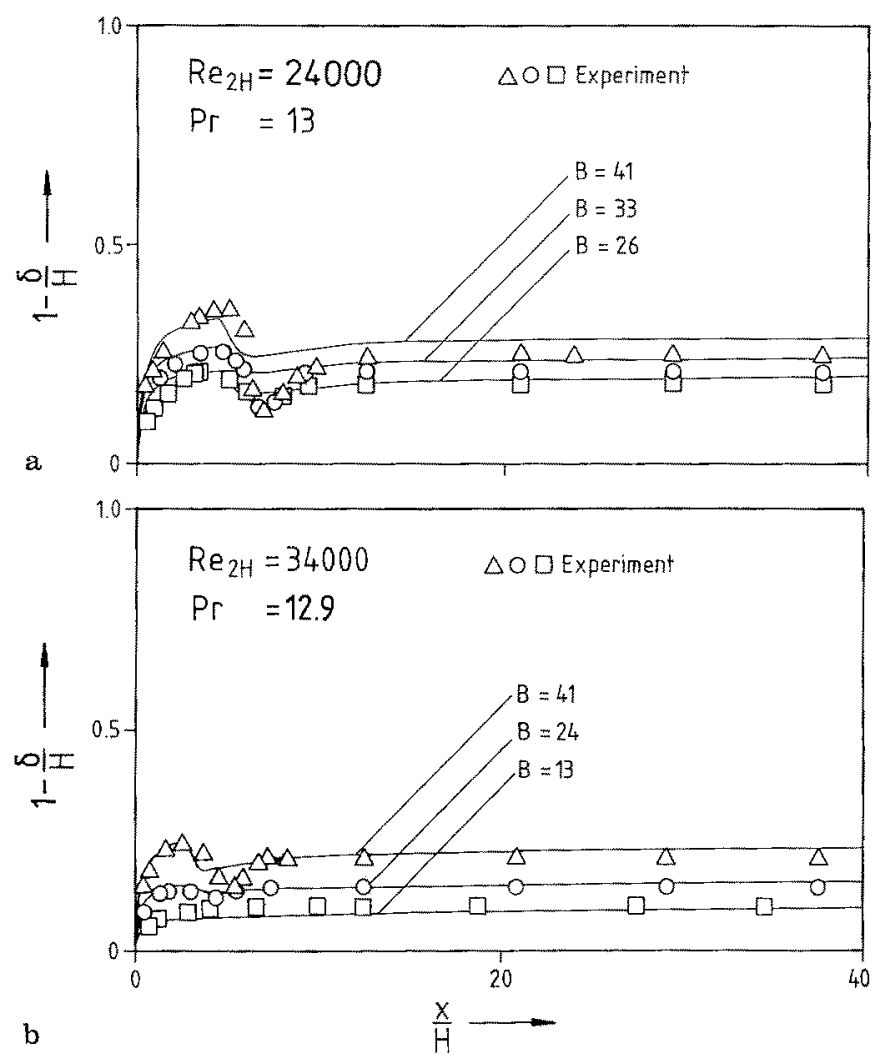

Fig. 7. Influence of the cooling parameter $B$ on the shape of the frozen crust (wavy ice layer)

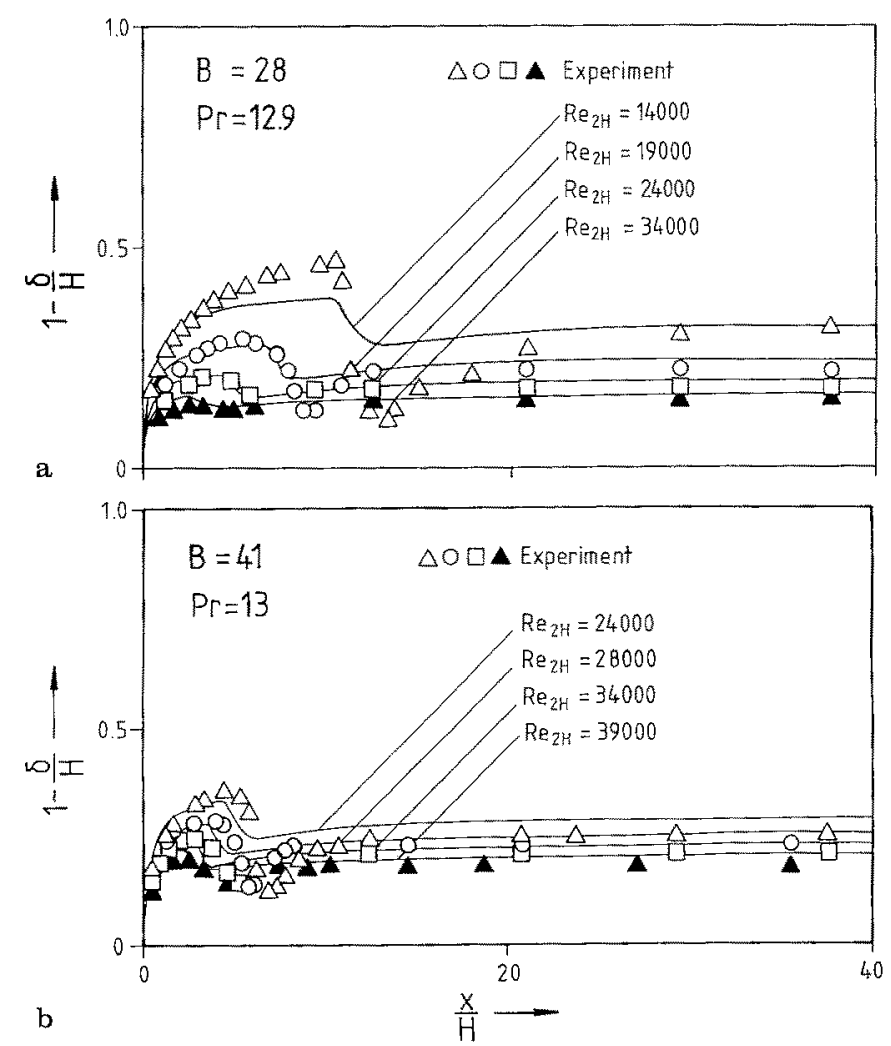

Fig. 8. Influence of the Reynolds number on the shape of the frozen crust (wavy ice layer) 


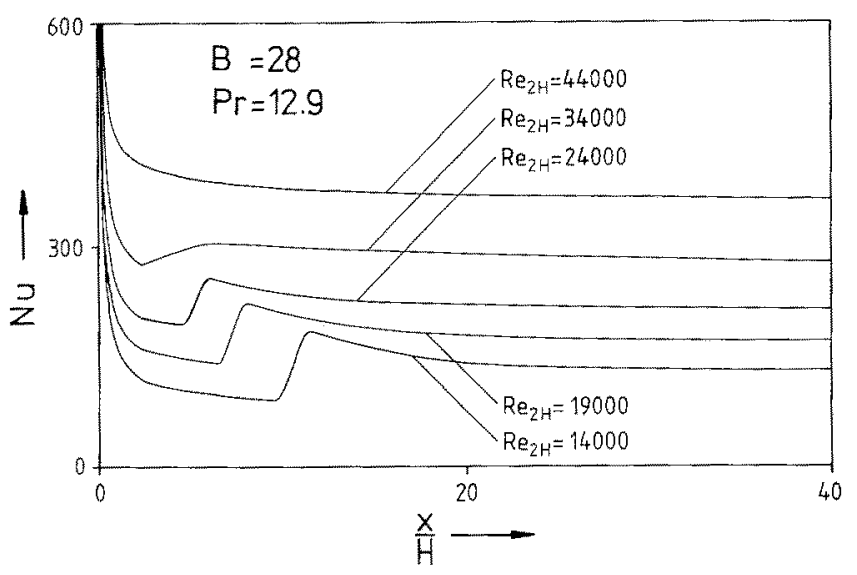

Fig. 9. Nusselt number as a function of $x / H$ with $R e_{2 H}$ as parameter

For the ice layers depicted in Fig. 8, the following Fig. 9 shows the variation of the local Nusselt number which is defined by

$N u=\frac{\left.2 \delta \frac{\partial T}{\partial y}\right|_{y=\delta}}{T_{\mathrm{F}}-T_{\mathrm{b}}}$

where $T_{\mathrm{b}}$ is the bulk temperature of the fluid. As can be seen, the Nusselt number decreases monotonously for $R e_{2 H}=$ 44,000 , indicating the existence of a smooth ice layer for the prescribed parameters. With decreasing Reynolds number for the given value of $B$, a small wave develops near the entrance of the cooling section (Fig. 8). This results in an increasing value of the Nusselt number for $\tilde{x}>\tilde{x}_{\mathrm{tr}}$. For a further decrease of the Reynolds number with $B=$ const., the increase in the Nusselt number in the diffusor region of the flow is more pronounced. After reaching the maximum value in the diffusor region, the Nusselt number decreases monotonously in axial direction. The limiting value for $N u$, which is reached for large axial distances, is higher than the value of $N u$ at $\tilde{x}=\tilde{x}_{\mathrm{tr}}$, indicating the retransition of the laminarized flow.

\section{Conclusions}

According to the experimental and theoretical results of the present investigation concerning the freezing of water flow between asymmetrically cooled parallel plates, the following major conclusions may be drawn

1. A simple numerical model was presented for calculating smooth ice layers for laminar- and turbulent flow in an asymmetrically cooled channel.

2. An approximation method was used for predicting smooth ice layers for laminar- and turbulent flow.
3. The numerical model of Weigand and Beer [14] was adopted for calculating wavy ice layers with one wave in the cooled channel. It was shown that the model is capable, after small changes, to deal with the case under consideration.

4. It was observed that a full laminarization of the flow takes place near the entrance of the test section for low values of the Reynolds number. Therefore, the mechanism which leads to the development of wavy ice layers with one wave is found to be quite similar to the one for a cooled flat plate for low values of the Reynolds number.

\section{References}

1. Zerkle, R. D.; Sunderland, J. E.: The effect of liquid solidification in a tube upon laminar-flow heat transfer and pressure drop. J. of Heat Transfer 90 (1968) 183-190

2. Lee, D. G.; Zerkle, R. D.: The effect of liquid solidification in a parallel plate channel upon laminar-flow heat transfer and pressure drop. J. of Heat Transfer 91 (1969) 583-585

3. Kikuchi, Y.; Shigemasa, Y; Oe, A.; Ogata, T.: Steady state freezing of liquids in laminar flow between two parallel plates. J. Nucl. Sci. Technol. 23 (1986) 43-55

4. Weigand, B.; Beer, H.: Liquid solidification in a parallel place channel upon laminar-flow heat transfer: The stationary case. Wärme- und Stoffübertrag. 26 (1991) 233-240

5. Cheng, K. C.; Sum-Lok Wong: Asymmetric solidification of flowing liquid in a convectively cooled parallel-plate channel. Appl. Sci. Res. 33 (1977) 309-335

6. Cho, C.; Özisik, M.: Transient freezing of liquids in turbulent flow inside tubes. J. Heat Transfer 101 (1979) 456-468

7. Shibani, A.; Özisik, M.: A solution of freezing of liquids of low Prandt number in turbulent flow between parallel plates. J. Heat Transfer 99 (1977) 20-24

8. Weigand, B.; Beer, H.: Freezing in turbulent flow inside tubes and channels. Wärme- und Stoffübertrag. 28 (1993) 57-64

9. Gilpin, R. R.: The morphology of ice structure in a pipe at or near transition Reynolds numbers. Heat Transfer - San Diego 1979, AICHE Symp. Ser. 189 (1979) 89-94

10. Gilpin, R. R.: Ice formation in a pipe containing flows in the transition and turbulent regimes. J. Heat Transfer 103 (1981) 363-368

11. Gilpin, R. R.; Hirata, T.; Cheng, K. C.: Wave formation and heat transfer at an ice-water interface in the presence of a turbulent flow. J. Fluid Mech. 99 (1980) $619-640$

12. Seki, N.; Fukusako, S.; Younan, G. W.: Ice formation phenomena for water flow between two cooled parallel plates. J. Heat Transfer 106 (1984) 498-505

13. Weigand, B.; Beer, H.: The morphology of ice structure in a parallel plate channel. Proc. 3rd Int. Symp. on Cold Regions Heat Transfer, Fairbanks, Alaska, USA (1991) 167-176

14. Weigand, B.; Beer, H.: Ice formation phenomena for water flow inside a cooled parallel plate channel: an experimental- and theoretical investigation of wavy ice layers. Int. J. Heat Mass Transfer 36 (1993) 685-693

15. Tago, M.; Fukusako, S; Yamada, M.; Horibe, A.; Okagaki, O.: An experimental study of ice layer transition phenomena observed in a duct containing water flow. Proc. 3rd Int. Symp. on Cold Regions Heat Transfer, Fairbanks, Alaska, USA (1991) $257-269$

16. Weigand, B.; Beer, H.: Solidification of flow liquid in an asymmetric cooled parallel-plate channel. Int. Comm. Heat Mass Transfer 19 (1992) 17-27 
17. Moretti, P. M.; Kays, W. M.: Heat transfer to a turbulent boundary layer with varying free-stream velocity and varying surface temperature - an experimental study. Int. J. Heat Mass Transfer 8 (1965) 1187-1202

18. Cebeci, T.; Chang, K. C.: A general method for calculating momentum and heat transfer in laminar and turbulent duct flows. Numer. Heat Transfer 1 (1978) 39-68

19. Reyhner, T. A.; Flügge-Lotz, I.: The interaction of a shock wave with a laminar boundary layer. Int. J. Nonlinear Mech. 3 (1968) $173-199$

20. Kwon, O. K.; Pletcher, R. H.; Lewis, J. P.: Prediction of sudden expansion flows using the boundary-layer equations. J. Fluid Engng. 106 (1984) 285-291
Prof. Dr.-Ing. H. Beer

Institut für Technische Thermodynamik.

Petersenstraße 30

64287 Darmstadt/Germany

Dr.-Ing. B. Weigand

ABB Power Generation Ltd.

Gas Turbine Development

$\mathrm{CH}-5401$ Baden/Switzerland

Received on January 13, 1993 